www.jmscr.igmpublication.org

Impact Factor 5.84

Index Copernicus Value: 83.27

ISSN (e)-2347-176x ISSN (p) 2455-0450

crossref DOI: _https://dx.doi.org/10.18535/jmscr/v5i7.74

Journal Of Medical Science And Clinical Research

IGM Publication

An official Publication of IGM Publication

\title{
A Comparative study of Topical Besifloxacin Vs. Moxifloxacin in Management of Bacterial Keratitis
}

\author{
Authors \\ Dr Subhabrata Parida, Assoc Prof, Dr Satya Ranjan Mallick \\ Department of Ophthalmology, SCB Medical College, Cuttack, Odisha, India \\ Corresponding Author \\ Dr Subhabrata Parida \\ Associate Professor, Ophthalmology, SCB Medical College, Cuttack \\ Ph: 9937041560, Email: Subhabrataparida@yahoo.co.uk
}

\begin{abstract}
This comprehensive review summarises the pharmacokinetics, efficacy, and safety of besifloxacin ophthalmic suspension $0.6 \%$ against moxifloxacin $0.3 \%$ solution and examines its role in the treatment of ocular surface bacterial infections. Besifloxacin possesses balanced activity against bacterial topoisomerase 2(also called DNA gyrase) and topoisomerase 4. Ocular pharmacokinetic studies have shown that besifloxacin achieves high, sustained concentrations in the tear fluid and conjunctiva following topical administration, with negligible systemic exposure. Large randomized, controlled clinical trials have established the efficacy and safety of besifloxacin administered 3 times daily for 5 days for treatment of acute bacterial conjunctivitis in both adults and children.

In our study, we are trying to compare the efficacy of besifloxacin vs. moxifloxacin topical for simple bacterial keratitis without perforation or any other complication. Patients with other systemic diseases like diabetes, collagen diseases, immunocompromised state or any other infectious disease were excluded from our study. All culture positive acute bacterial keratitis coming to our tertiary institute were selected randomly. Alternatively we treated them with either besifloxacin or moxifloxacin. Then followed them on 3,7,15,30 days respectively, noting all signs, symptoms, slitlamp biomicroscopy, visual acuity, slitlamp photography, patient's overall satisfaction etc.

We found both the drugs equally effective, but doses frequency less in besifloxacin group, patient reliability and comfort also more in besifloxacin group.

Keywords- Topical, Besifloxacin, Moxifloxacin, Bacterial Keratitis.
\end{abstract}

\section{INTRODUCTION}

Bacterial keratitis is an infectious disease that involves cornea and can lead to significant visual loss. Infectious process involving cornea producing keratitis that may be mild or severe, require an early and vigorous treatment with an effective antimicrobial therapy, it can minimise corneal scaring and visual loss. ${ }^{1}$ Various treatment regimen include frequent administration of topical antibiotics often at higher concentration. Multiple use of antibiotics, simultaneously may lead to toxicity. Fluoroquinolones changed the paradigm of the treatment of bacterial keratitis. ${ }^{2}$ Monotherapy with fluoroquinolones can be used 
for bacterial keratitis. Most common organism that causes Bacterial keratitis are S. aureus, $\mathrm{S}$ pneumonae, pseudomonas and other gram negative bacilli. ${ }^{3}$

Fluoroquinolones after advantages of good ocular penetration and broad spectrum efficacy, good safety profile, Moxifloxacin is a fourth generation fluoroquinolones it exhibit a broad spectrum bactericidal activity against both gram -ve and gram +ve bacilli. Moxifloxacin has superior corneal scaring and gives penetration. ${ }^{4}$

Besifloxacin is another topical antibiotics that can be used as an option to treat bacterial keratitis. It is a fluroquinolones that has broad spectrum antibiotic activity. It is a FDA approved drug for that treatment of bacterial keratitis. It has shown success in treating Bacterial keratitis as well as present complication like scaring. ${ }^{5}$

\section{MATERIALS AND METHODS}

A retrospective case analysis of 80 patients with Corneal Ulcer treated ata tertiary eye care centre, from March 2016 to April 2017, was carried out. The patients were divided into twogroups of 40 patients each. Group-A patients were treated with Besifloxacin and group-B patients were treated with Moxifloxacin. As soon as the diagnosis of Bacterial Keratitis was made, group-A patients were started on Besifloxacin 4 hourly for 3 days.. Similarly after making a diagnosis of bacterial keratitis group-B patients were administered Moxifloxacin solution 4hourlya day for first 03 days. The therapy was continued for at least 03 weeks or at least a week after resolution of all clinical signs and symptoms and then tapered off. The response to the two regimens was evaluated and compared at day 3, 7, 15 and 30. At each visit the patients were examined on slit-lamp,iop and cultuture sensitivity was done. Assesment include clinical evaluation of signs and symptoms, VA, S/L biomicroscopy and culture of the infected eye at each visit as well as indirect ophthalmoscopy, slit lamp photography were also done.

\section{RESULTS}

A total of 80 patients (all 80 with culture confirmed bacterial keratitis) were randomised. Based on the $95 \%$ confidence interval of the difference, besifloxacin was non inferior to moxifloxacin for clinical resolution on day 5 (3\% vs $4 \%$ respectively) and Day 8 (84.5\% vs $84.0 \%)$. and for microbial eradication on day $5(9.3 \%$ vs $91.1 \%$ ). There was no statistically significant difference between the 2 treatment groups for either efficacy end point on day 5 or $8(\mathrm{p}>0.05)$. Besifloxacin and Moxifloxacin were well tolerated. The cumulative frequency of ocular adverse events was similar between treatment $(12 \%$ and $14 \%$ with besifloxacin and moxifloxacin respectively). However, eye irritation occurred more often in moxifloxacin treated eyes $(0.3 \%$ for besifloxacin vs $1.4 \%$ for moxifloxacin, $\mathrm{p}=0.0201$ ).

\section{DISCUSSION}

Bacterial keratitis, an infection of cornea, has potential for serious complications, including corneal scarring and permanent visual impairement or vision loss, if treatment is delayed. ${ }^{6}$ Common keratitis-causing pathogens include $\mathrm{S}$. aureus, P.aerugenosa, S.pneumonae and serratia species. $^{7}$ The American Academy of Ophthalmology issued guidelines recommending topical broad-spectrum antibiotics for empirical treatment of bacterial keratitis. With regard to the treatment of bacterial corneal ulcers, topical fluoroquinolones are considered the drug of choice in absence of microbiological evaluation. ${ }^{8}$

A large, retrospective, case surveillance study showed that besifloxacin was as safe as moxifloxacin in the treatment of bacterial keratitis, with similar low incidences of corneal scarring and neovascularisation, and similar rates of physician-assesed bacterial eradication. ${ }^{9}$ Along with the efficacy demonstrated by besifloxacin against S.aureus, P.aerugenosa, and serratia species in patients with bacterial conjunctivitis. These results suggest a potential role for besifloxacin in the treatment of bacterial keratitis. 
However, randomized, multicentric, large number, controlled clinical trials of besifloxacin in the treatment of bacterial keratitis are needed.

\section{CONCLUSION}

Besifloxacin ophthalmic suspension was not inferior to moxifloxacin ophthalmic suspension and provided similar safety and effiicacy (clinical and microbiological) outcomes when used for treatment of bacterial keratitis. Patients found to be more reliable for besifloxacin for its less frequent doses and less irritation. As it's a new drug, resistance is also almost nil. It's a very good option to treat bacterial conjunctivitis and keratitis in both adults and children. But a more extensive, multicentric studies still required to find more effects of besifloxacin.

\section{REFERENCES}

1. Carter NJ, Scott LJ. Besioxacin ophthalmic suspension 0.6\%. Drugs. 2010;70:83-97

2. McDonnell PJ. How do general practitioners manage eye diseases in the community? $\mathrm{Br} \quad J \quad$ Ophthalmol. 1988;72:733-6

3. O'brien TP. Besioxacin ophthalmic suspension, $0.6 \%$ : a novel topical furoroquinolone for bacterial conjunctivitis. Adv Ther. 2012;29:473-90.

4. Carreras B. Bacteriological analysis in the management of conjunctivitis. Comparison of antibiotic resistance between 1982 and 2008. Arch Soc Exp Oftalmol. 2012;87:107-11

5. Wong RL, Gangwani RA, Yu LW, Lai JS. New treatments for bacterial keratitis. $J$ Ophthalmol. 2012;2012:831502

6. Hwang DG. Fluoroquinolone resistance in ophthalmology and the potential role for newer ophthalmic fluoroquinolones. Surv Ophthalmol. 2004:49(Suppl. 2):S79-83
7. DeLeon J. Silverstein BE, Allaire C, et al. Besioxacin ophthalmic suspension $0.6 \%$ administered twice daily for 3 days in the treatment of bacterial conjunctivitis in adults and children. Clin DrugInvestig. 2012; 32:303-17.

8. American Academy of Ophthalmology Cornea/External Disease Panel. Preferred Practice PatternGuidelines. Bacterial Keratitis. San Francisco, Calif.: American Academy of Ophthalmology; 2013.

9. Schechter BA, Parekh JG, Trattler W. Besioxacin ophthalmic suspension, $0.6 \%$ in the treatment of bacterial keratitis: a retro-spective surveillance study. $J$ Ocul Pharmacol Ther. 2015;31(2):114-21. 